\title{
Mobilization Clinical Trial Setting
}

National Cancer Institute

\section{Source}

National Cancer Institute. Mobilization Clinical Trial Setting. NCI Thesaurus. Code C156596.

A clinical trial or treatment setting in which the therapy of interest is considered mobilization therapy. 